\title{
Introduction to Chern-Simons Theories
}

\author{
Jorge Zanelli*† \\ Centro de Estudios Científicos (CECS), Valdivia, Chile \\ E-mail: z-at-cecs.cl
}

\section{Luis Huerta}

Departamento de Ciencias Aplicadas, Facultad de Ingeniería, Universidad de Talca

E-mail: Ihuerta-at-aac.cl

Chern-Simons theories occur in Physics more often than one may think. In particular, the Hamiltonian action for any mechanical system with finite number of degrees of freedom is a CS Lagrangian. CS forms can be seen as generalizations of the coupling between the electromagnetic field and a point charge. It should come as no surprise, therefore, that the CS forms are perfectly suited to describe the consistent coupling of a (nonabelian) gauge connection to extended charged objects (branes). An application of this is provided by a spacetime region $M$ where the electromagnetic field is described by the Lagrangian $\frac{1}{4}\left(F \wedge^{*} F+\theta F \wedge F\right)$. Since the Maxwell equations are unaffected by the presence of the $\theta$-term, this represents a slight modification of the vacuum in $M$ ( $\theta$-vacuum). This modification is shown to correspond to the addition of a form of matter that couples through a CS form on the surface of $M$. The connection to superconductivity is also briefly mentioned.

4th International Conference on Fundamental Interactions -ICFI2010,

August 1-7, 2010

Viçosa, Brazil

* Speaker.

$\dagger$ A footnote may follow. 


\section{Introduction}

Chern-Simons (CS) forms provide Lagrangians for gauge theories, invariant under some symmetry group $\mathbb{G}$ in a certain odd-dimensional manifold $M$. The fundamental object in a gauge theory is the gauge connection A (known as the vector potential in physics). If $g(x) \in \mathbb{G}$ is an element of the group acting independently at each point in spacetime, the connection transforms as

$$
\mathbf{A} \rightarrow \mathbf{A}^{\prime}=g \mathbf{A} g^{-1}+g d\left(g^{-1}\right) .
$$

The connection is gauge-dependent and, therefore, not directly measurable. However, the curvature (field strength in physics), $\mathbf{F}=d \mathbf{A}+\mathbf{A} \wedge \mathbf{A}$, transforms in a tensorial representation of the gauge group,

$$
\mathbf{F} \rightarrow \mathbf{F}^{\prime}=g \mathbf{F} g^{-1},
$$

and has more direct observable features. The four fundamental forces of nature are described by gauge theories. The standard model of electromagnetic, weak and strong interactions, is a triumph of the gauge principle, spanning $10^{10}$ orders of magnitude in energy, from the scale of the chemical bond to the physics at the LHC, and possibly beyond. General Relativity, albeit a classical theory, is founded on the equivalence principle, which states the invariance of the laws of physics under a gauge symmetry: local Lorentz transformations. Moreover, Einstein identified the gauge field associated to this invariance as the gravitational interaction, which makes him the discoverer of the first nonabelian gauge theory, several decades prior to Yang and Mills.

Chern Simons forms can be understood as lagrangian densities for some gauge potential in odd-dimensional spacetimes. Mathematically, CS forms are related to topological structures like the Euler or the Pontryagin invariants. If we denote by $P_{2 k}(\mathbf{F})$ one of those invariants, then $P_{2 k}$ satisfies the following conditions [1]:

i. It is a polynomial in the curvature $\mathbf{F}$ associated to a gauge connection $\mathbf{A}$.

ii. It is invariant under gauge transformations (1.1) and (1.2).

iii. It is closed, $d P_{2 k}=0$.

iv. It can be locally expressed as the derivative of a $(2 k-1)$-form, $P_{2 k}=d \mathscr{C}_{2 k-1}$.

v. Its integral over a $2 k$-dimensional compact, orientable manifold without boundary, is a topological invariant, $\int_{M} P_{2 k}=c_{2 k}(M) \in \mathbf{Z}$.

Condition (ii) is satisfied if $P_{2 k}$ is a trace over the gauge algebra of a product of curvature 2 -forms. Condition (iii) is a consequence of the Bianchi identity which states that the covariant derivative (in the connection $\mathbf{A}$ ) of the curvature $\mathbf{F}$ vanishes identically: $D \mathbf{F}=d \mathbf{F}+[\mathbf{A}, \mathbf{F}] \equiv 0$. Condition (iv) follows from (iii) as a direct consequence of Poincare's lemma: If $D \phi=0$ then $\phi=d$ (something). The forms $\mathscr{C}_{2 n-1}$ are the Chern-Simons forms and are the subject of these notes. Finally, (v) means that, although in a local chart $P_{2 k}$ looks like an exact form, globally it is not.

The invariance of $P_{2 k}$ under gauge transformations is easily seen from the homogeneous transformation law for the curvature (1.2), which implies that $\operatorname{Tr}\left[\mathbf{F}^{k}\right]$ is invariant by construction, due to the cyclic property of the trace. This has a very useful consequence: under gauge transformations, CS forms change by an exact form (total derivative). This can be seen from combining features listed above. From (iv), a gauge transformation gives

$$
\delta_{\text {gauge }} P_{2 k}=d\left(\delta_{\text {gauge }} \mathscr{C}_{2 k-1}\right),
$$


and since $P_{2 k}$ is invariant, one concludes that

$$
d\left(\delta_{\text {gauge }} \mathscr{C}_{2 k-1}=0\right) .
$$

By Poincare's lemma, this last equation implies that the gauge variation of $\mathscr{C}_{2 k-1}$ can be written locally as an exact form,

$$
\delta_{\text {gauge }} \mathscr{C}_{2 k-1}=d \Omega .
$$

This is a nontrivial feature: although the nonabelian connection $\mathbf{A}$ transforms as in (1.1), the CS form transforms in the same way as an abelian connection, and this is sufficient to ensure that CS forms define gauge invariant actions. The reason is the same that makes the electromagnetic coupling between a point charge and its surrounding electromagnetic field, $I[A]=\int_{\Gamma} A$, gauge invariant:

$$
\delta_{\text {gauge }} I[A]=\int_{\Gamma} \delta_{\text {gauge }} A=\int_{\Gamma} d \Omega,
$$

which vanishes for any reasonable set of boundary conditions. Apart from this, CS actions are exceptional in physics because they do not require a metric structure in spacetime in order to construct Lorentz invariants, as is the case for most physical systems, like the Maxwell or YangMills theories. A particular consequence of this is that in CS gravitation theories the metric is a derived (composite) object and not a fundamental field to be quantized. This in turn implies that concepts such as the energy-momentum tensor and the inertial mass are phenomenological constructs of classical or semi-classical nature.

Another important difference with Yang-Mills systems is that CS lagrangians are functions of a connection $\mathbf{A}$ and its exterior derivatives that cannot be written as local functions involving only the curvature $\mathbf{F}$. A direct consequence of this is that CS forms are not necessarily defined globally throughout the spacetime manifold $M$, and many charts may be required to define them.

\subsection{Historical background}

CS forms were originally introduced in physics in the discussion of chiral anomalies, which signal the violation of the classically conserved chiral currents due to quantum mechanical corrections. By direct computation, the deviation from the classical conservation law for the chiral current (anomaly) in four spacetime dimensions, was shown to be proportional to the Chern class $P_{4}(\mathbf{F})[2,3]$. It was later observed that this form could be written as the exterior derivative of a local three form, $P_{4}(\mathbf{F})=d \mathscr{C}_{3}(\mathbf{A})$, where $\mathscr{C}_{3}(\mathbf{A})$ is a function of the connection, originaly discussed by Chern and Simons in the mathematical literature [4]. For a historical overview, see [5].

A CS form seems to have been used as a Lagrangian for the first time in the 11-dimensional supergravity model of Cremmer, Julia and Scherk. There, the action contains a CS term for a threeform field needed by supersymmetry [6]. It was later realized that CS forms define potentially useful field theory Lagrangians in three spacetime dimensions. CS actions have also been invoked for the description of the quantum Hall effect [7] and were shown to be related to polynomial invariants of knot theory [8]; CS theory is also closely related to conformal field theory and the Wess-Zumino-Witten (WZW) action in two dimensions [8, 9], and to superconductivity [10, 11]. Finally, the standard gravity theory in $2+1$ dimensions itself was shown to be a CS system [12, $13,14]$. Moreover, CS forms in more dimensions can describe gravities or supergravities which 
are genuine gauge theories [15] (for a review, see [16]). As discussed in the next subsections, however, examples of CS actions have been around much longer in the context of mechanics and electrodynamics.

\section{Examples of Chern-Simons theories}

The CS forms $\mathscr{C}_{2 k+1}(\mathbf{A})$ are defined by the label $k$ and the Lie algebra $\mathscr{G}$ associated to the gauge group $\mathbb{G}$. In this section we review some examples.

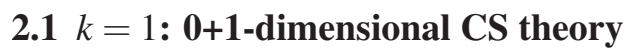

For $k=1$, the invariants $P_{2 k}$ take the rather simple form, with $\mathbf{F}=F^{a} \mathbf{J}_{a}$, where $\mathbf{J}_{a}$ are the generators of the Lie group $\mathbb{G}$. Since most gauge groups of physical interest, $S U(n, m), S O(n, m)$, etc. are of determinant one, their generators are traceless and therefore $\operatorname{Tr}[\mathbf{F}]=0$. There is only one very important exception to this rule: electrodynamics. In this case, the gauge group is $U(1)$ and the generator is the imaginary unit, $i$, so the trace can be dropped and the invariant is just the two-form which corresponds to the field strength,

$$
P_{2}=F=\frac{1}{2} F_{\mu v} d x^{\mu} \wedge d x^{v},
$$

and the respective CS form is the $U(1)$ connection

$$
\mathscr{C}_{1}=A=A_{\mu} d x^{\mu} .
$$

This CS form is relevant for the coupling of an electrically charged point particle with an external electromagnetic field,

$$
I=e \int_{\Gamma} A_{\mu}(z) d z^{\mu}
$$

which has the peculiar features indicated above. First, it does not make reference to the metric of the spacetime where the interaction takes place; it does not require the existence of a metric structure to make sense. Second, although it depends explicitly on a non-invariant gauge field, it is invariant under proper $^{1}$ gauge transformations.

This unique form of interaction accounts for the coupling between charged matter and the electromagnetic field, implemented through the substitution of ordinary derivatives by (gauge) covariant derivatives. This form of interaction in turn means that the field that mediates the electromagnetic interaction is a connection on a fiber bundle (gauge theory). This is also the simplest example of a CS system.

The action (2.3) can be viewed as a functional of $A$ or as a functional of the embedding coordinates $z^{\mu}$ that parametrize the trajectory of the charge in spacetime. In the first case, it might seem to lead to an inconsistent classical system: the Euler-Lagrange equation obtained varying with respect to $A$ reads $1=0$. However, this is a very narrow interpretation of the variation. In fact, what one obtains is

$$
\delta I[A]=e \int_{\Gamma} \delta A_{\mu}(z) d z^{\mu}=0,
$$

\footnotetext{
${ }^{1}$ Proper gauge transformations are those that approach the identity at infinity, $A^{\prime}=A+d \Omega$, so that at infinity, $\Omega \rightarrow$ [constant]. It can be seen that this condition can be replaced by the requirement that the spacetime manifold be bounded or periodic in time.
} 
which only states that the integrand must be an exact form,

$$
\delta A(z)=d \alpha(z),
$$

with $\alpha\left(z_{+}\right)=\alpha\left(z_{-}\right)$, where $\left\{z_{+}, z_{-}\right\}=\partial \Gamma$. In other words the classical equation only informs us of the fact that $A$ can change as an abelian connection, but it is otherwise arbitrary, and $\alpha(z)$ is periodic. The periodicity of $\alpha(z)$ can be automatically guaranteed if the manifold $\Gamma$ is closed on itself, i. e., if it has no boundary, $\partial \Gamma=0$. So, the action principle hints to the fact that the coordinate $z$ along $\Gamma$ should be periodic [17].

\subsection{Classical mechanics as a $0+1 \mathrm{CS}$ system}

In the alternative interpretation, equation (2.3) is considered as a functional of $z^{\mu}$, the embedding coordinates for $\Gamma$ in a higher-dimensional target manifold. This is the conventional interpretation leading to equations of motion for a charge in the presence of an external e-m potential $A$, although here we have not included a kinetic term for the charge $\left(K=\frac{1}{2} \gamma_{a b}(z) \dot{z}^{a} \dot{z}^{b}\right)$, which requires a spacetime metric. However, as we will show next, this is not really necessary.

Consider the action for a mechanical system of a finite number of degrees of freedom in Hamiltonian form,

$$
I[p, q]=\int_{\Gamma}\left[p_{i} d q^{i}-H(p, q) d t\right], \quad i=1,2,3, \ldots, s,
$$

which can be seen to be of the form (2.3) if one identifies $A_{i}=p_{i}, A_{s+i}=0, A_{0}=-H$, and $z^{\mu}=$ $\left(t, q^{i}, p_{j}\right)$. In other words, the Hamiltonian action for any mechanical system is a (0+1)-CS form defined on the 1-dimensional history of the system in a $(2 s+1)$-dimensional embedding space $M^{2 s+1}$. Clearly, this embedding spacetime is just the phase space to which we have added the time.

The electromagnetic analogy can be made more apparent if the kinetic term is written in the skew-symmetric form, $\left(p_{i} d q^{i}-q^{i} d p_{i}\right) / 2$ (the corresponding identification is now $A_{i}=p_{i} / 2, A_{s+i}=$ $-q^{i} / 2$ ) [18]. Note that the phase space, enlarged by the inclusion of time, is odd-dimensional, and Hamilton's equations can be written as

$$
F_{a b} \dot{z}^{b}=E_{a}, \quad a, b=1,2,3, \ldots 2 s,
$$

where we have defined $E_{a}=F_{0 a}=\partial_{0} A_{a}-\partial_{a} A_{0}=\partial_{a} H$, and the field strength $F_{a b}$ is identified as the symplectic form. Thus, we conclude that all of classical mechanics can be understood as a CS system where Hamilton's equations describe a particle moving under the influence of an external electromagnetic field that produces zero net Lorentz force on the charge $(e[\vec{E}+\vec{v} \times \vec{B}]=0)$. One application of the analogy between the dynamics of classical mechanics and electromagnetic phenomena is Feynman's derivation of Maxwell's equations from classical mechanics [19].

Where is gauge invariance in a mechanical system? In this language, gauge invariance is just the invariance of the Euler-Lagrange equations under the addition of a total derivative to the Lagrangian, $L \rightarrow L+d \Omega(p, q, t)$, where $L=p_{i} d q^{i}-H(p, q) d t$. Thus, in this framework, gauge invariance is the symmetry of classical mechanics under canonical transformations.

\section{$2.3 k=2:$ CS field theory}

The next example of invariant form is

$$
P_{4}=\langle\mathbf{F} \wedge \mathbf{F}\rangle
$$


and the corresponding CS lagrangian is the three-form

$$
\mathscr{C}_{3}(\mathbf{A})=\left\langle\mathbf{A} \wedge d \mathbf{A}+\frac{2}{3} \mathbf{A} \wedge \mathbf{A} \wedge \mathbf{A}\right\rangle
$$

Here $\langle\cdots\rangle$ denotes the symmetrized $\operatorname{trace}^{2}$ over $\mathscr{G}$, the Lie algebra associated generated by $\left\{\mathbf{J}_{a}\right\}$. It can be checked directly that $d \mathscr{C}_{3}=\langle\mathbf{F} \wedge \mathbf{F}\rangle$.

Now there is no need to restrict ourselves to an abelian gauge group since the commutators $\operatorname{Tr}\left[\mathbf{J}_{a} \mathbf{J}_{b}\right]$ don't vanish in general. The action that corresponds to (2.3) now reads

$$
I[\mathbf{A}]=e \int_{\Gamma^{3}}\left\langle\mathbf{A} \wedge d \mathbf{A}+\frac{2}{3} \mathbf{A} \wedge \mathbf{A} \wedge \mathbf{A}\right\rangle,
$$

where $\Gamma^{3}$ denotes a three-dimensional world volume swept by the evolution of a 2-dimensional membrane. Since the Lagrangian now involves derivatives of the connection, extremizing the action under variations of $\mathbf{A}$ yields dynamical equations over $\Gamma^{3}$ for this field. That wasn't the case in the preceding section, where $A(z)$ was a prescribed, non dynamical function (an external potential).

Varying the CS action with respect to A produces -rather simple but non trivial- field equations,

$$
\left\langle\mathbf{J}_{a} \mathbf{F}\right\rangle=0,
$$

which in turn implies $\mathbf{F}=0$. These equations mean that on any open patch, the connection is flat and can be locally written as a pure gauge,

$$
\mathbf{A}(z)=g^{-1} d g,
$$

where $g(z)$ is any application from the worldvolume onto the gauge group, $g: \Gamma^{3} \rightarrow \mathbb{G}$. This means in particular, that there are no propagating degrees of freedom in this theory, since any configuration is locally equivalent to a flat connection $\mathbf{A}=0$, and can be gauged away. However, a locally flat connection doesn't necessarily describe a trivial situation. In fact, there exist many locally flat but topologically nontrivial configurations, as for example in the case of AdS gravity in $2+1$ dimensions, where the CS action admits locally flat classical solutions that describe interesting configurations such as black holes [20,21].

The three-dimensional CS field theories have been extensively studied in the past three decades, which makes it unnecessary to delve much further into this topic in an introductory survey. The reader can find a wealth of material in modern texts on field theory, such as [22, 23, 24]

The invariant $P_{4}=\langle\mathbf{F} \wedge \mathbf{F}\rangle$ has an interesting history of its own. This is the Pontryagin invariant that defines a characteristic class in the fibre bundle associated to a connection $\mathbf{A}$. In quantum field theory it appears as the obstruction to chiral current conservation (chiral anomaly), and as the Atiyah-Singer index associated to a Dirac spinor coupled to a Yang Mills field.

The integral of $P_{4}$ is a topological invariant or just a boundary term and therefore, if added to the lagrangian, it does not affect the field equations. Nevertheless, all topological densities like this change the value of the action, giving different weights in the quantum path integral to

\footnotetext{
${ }^{2} \mathrm{~A}$ note of caution is in order here: The symmetrized trace $\langle\cdots\rangle$ is a two-entry object, $\langle\rangle:, \mathscr{G} \times \mathscr{G} \rightarrow \mathbf{R}$. So, the expression $\langle\mathbf{A} \wedge \mathbf{A} \wedge \mathbf{A}\rangle$ actually stands for $1 / 2 A^{a} \wedge A^{b} \wedge A^{c}\left\langle\mathbf{J}_{a},\left[\mathbf{J}_{b}, \mathbf{J}_{c}\right]\right\rangle$, where $\mathbf{J}_{a}$ are the generators of the Lie algebra $\mathscr{G}$. Naturally, if $\mathscr{G}$ is abelian this term vanishes identically.
} 
configurations in different topological sectors. This in turn implies that the quantum vacuum of the theory is degenerate, containing a huge number of distinct zero-energy states among which the system can tunnel. Such a degenerate ground state is often called a $\theta$-vacuum, because the addition of $P_{4}$ to the action is with a coupling constant $\theta$.

In electromagnetism $P_{4}$ is a curiosity: it is the only Lorentz invariant bilinear in $F$, apart from the Maxwell Lagrangian, $F^{\mu v} F_{\mu \nu}$. It is gauge naturally invariant, but its addition does not modify Maxwell's equations, so it seems to play no role in classical electrodynamics. In the next section we will argue that that is not necessarily the case.

The abelian CS three-form looks like (2.9), but without the cubic term. This CS form should couple to a two-dimensional membrane whose world volume is a three-dimensional spacetime $\Gamma^{3}$,

$$
I[A]=\int_{\Gamma^{3}} A \wedge d A,
$$

whose classical equations are $F=0$, which means that locally, the space is free of electric and magnetic fields. Although this looks like a trivial situation, it is far from it. One could recall the Aharonov-Bohm experiment, which underscores the importance of nontrivial topology that makes a seemingly trivial configuration $(\vec{E}=\vec{B}=0)$ into a remarkable proof of nonlocality in quantum mechanics. This system is also related to other important applications such as superconductivity and the Hall effect. We will come back to this case in the next section.

\section{$2.4 k \geq 2$ : Higher dimensional CS theories}

CS field theories can be constructed for all odd dimensions in correspondence with the integral topological invariants (characteristic classes) of the dimension immediately above. The main difference between the different theories stem from the different Lie groups that are used. For $S U(N)$ groups, the topological invariants are the Pontryagin classes. For $S O(N)$ groups, besides the Pontryagin classes, there exists the Euler family. The corresponding CS actions give rise to the Lorentz CS theories in the first case, and to special forms of Lovelock actions in the second case [25]

\subsubsection{Generic case $(S U(N))$}

The extension of CS theories to higher dimensions is straightforward but nontrivial. The generalization is achieved by looking for the $(2 k-1)$-forms whose exterior derivative yields a Chern class for a given Lie-algebra valued connection in $(2 k)$ dimensions. The construction is rather unambiguous and only requires identifying an appropriate $k$ th rank invariant tensor $\tau_{a_{1} a_{2} \cdots a_{k}}:=$ $\left\langle\mathbf{J}_{a_{1}} \mathbf{J}_{a_{2}} \cdots \mathbf{J}_{a_{k}}\right\rangle$, where $\langle\cdots\rangle$ is the symmetrized trace over the Lie algebra. Then, the CS lagrangian form reads

$$
\mathscr{C}_{2 k-1}[\mathbf{A}]=\left\langle\mathbf{A} \wedge(d \mathbf{A})^{k-1}+\alpha_{1} \mathbf{A}^{3} \wedge(d \mathbf{A})^{k-2}+\cdots+\alpha_{k-1} \mathbf{A}^{2 k-1}\right\rangle,
$$

where $\left\{\alpha_{1}, \cdots \alpha_{k-1}\right\}$ are fixed rational numbers.

CS theories for dimensions $D \geq 5$ have been studied in different contexts (see e. g., [26, 27]), and in contrast with the case $D=3$, they are not necessarily topological and contain propagating local degrees of freedom [28].

These systems, however, exhibit pathological features, which obscure the analysis of their dynamical nature. First, the rank of the symplectic form is generically not constant throughout phase 
space, becoming noninvertible on some surfaces, $\Sigma$. In this case the system evolves from an initial state into another with fewer degrees o freedom in a finite time [18]. Second, the symmetry generators (first class constraints) may become functionally dependent in some subsets of phase space. There, the canonical analysis breaks down and it is no longer clear how to identify the physical observables (propagating degrees of freedom, conserved charges, etc.) and Dirac's canonical formalism [29] cannot be applied. Fortunately, the troublesome configurations generically occur in sets of zero measure in phase space and one can always restrict the attention to open regions where the canonical analysis holds (canonical regions) [30, 31].

\subsubsection{CS gravity $(S O(N))$}

It was observed by Witten that three-dimensional gravity can be cast as a CS theory for the Poincaré, de Sitter or anti-de Sitter groups, depending on the value of the cosmological constant $(\Lambda=0, \Lambda>0$ or $\Lambda<0)$ [14]. It turns out that this continues to be true for any odd dimension[15]. Moreover, the supersymmetric extension of these theories is quite elegant and yields a family of very interesting supergravities in which the spacetime symmetries (rotations, Lorentz boosts, translations or AdS bosts) are part of the gauge superalgebra [32,33]. The construction that leads to this conclusion is quite interesting, but lies somewhat outside the scope of this note. The reader can refer to [16] and references therein for a review of this topic.

The most appealing feature of CS gravity is that it is a bona fide gauge theory, constructed using only a connection in which the Lorentz ("spin") connection and the vielbein are combined. As a consequence, the metric does not appear as a fundamental field in the action principle, but as a derived entity in the solutions. In this way, the gravitational field is described by more degrees of freedom than those encoded in the metric alone, which is viewed as a composite emerging feature of the full geometrical content of spacetime. Other appealing features of CS gravities is its absence of dimensional coupling constants: as in any CS theory all coefficients appearing in the action are fixed rational numbers that cannot be renormalized without breaking gauge invariance. As a consequence, the corresponding quantum theory is expected to be scale invariant and finite.

The main difficulty of this elegant structure is how to couple them to external sources. The naive coupling of brane appropriately charged to contract its world and group indices to the connection usually leads to inconsistencies. This problem was detected in the attempts to couple an extended object with a nonabelian $p$-form that would play the role of a connection [34]. This difficulty was encountered again in the context of CS supergravity [35]. The problem is in essence, that a coupling of the form $\mathbf{j} \cdot \mathbf{A}$, where $\mathbf{j}$ is an extended source, typically breaks gauge invariance.

\section{Extended sources and CS couplings}

We observe that a $(2 n+1)$-CS form describes the coupling between a connection $\mathbf{A}$ and a membrane whose time evolution sweeps a $(2 n+1)$-dimensional volume. The consistency of this scheme follows from the precise form of the coupling [36],

$$
I[\mathbf{A} ; \mathbf{j}]=\int\left\langle\mathbf{j}_{2 p} \wedge \mathfrak{C}_{2 p+1}(\mathbf{A})\right\rangle,
$$

where $\mathscr{C}_{2 p+1}$ is the CS $(2 p+1)$-form living on the brane history, and we have defined $\mathscr{C}_{2 p+1}=$ $\left\langle\mathfrak{C}_{2 p+1}(\mathbf{A})\right\rangle$. The current generated by the $2 P$-brane is represented by the $(D-2 p-1)$-form $\mathbf{j}$ 
supported on the $(2 p+1)$-dimensional worldvolume $\Gamma^{2 p+1}$ of the brane,

$$
\mathbf{j}_{2 p}=q j^{a_{1} a_{2} \cdots a_{s}} \mathbf{J}_{a_{1}} \mathbf{J}_{a_{2}} \cdots \mathbf{J}_{a_{s}} \boldsymbol{\delta}(\Gamma) d x^{\alpha_{1}} \wedge d x^{\alpha_{2}} \wedge \cdots d x^{\alpha_{D-2 p-1}},
$$

where $d x^{\alpha_{i}}$ are transverse directions to $\Gamma$. The integration over the $D-2 p-1$ transverse directions yields a $(2 p-1)$-CS form integrated over the worldvolume of the $2 p$-brane. This integral might vanish -for instance, if $\left\langle\mathfrak{C}_{2 p+1}\right\rangle$ is zero for the corresponding gauge algebra. The fact that the current is supported on a submanifold $(\Gamma)$ of the entire spacetime $(M)$ implies that on the complement of $\Gamma$ the system enjoys full invariance under the entire gauge group. On $\Gamma$, however, the gauge symmetry is reduced to the subgroup that commutes with $K=j^{a_{1} a_{2} \cdots a_{s}} \mathbf{J}_{a_{1}} \mathbf{J}_{a_{2}} \cdots \mathbf{J}_{a_{s}}$.

It can be checked directly, using the symmetry properties of the bracket $\langle\cdots\rangle$, that under a gauge transformation, $I[\mathbf{A} ; \mathbf{j}]$ changes by a (locally) exact form, provided the current is covariantly conserved,

$$
D \mathbf{j}_{2 p}=d \mathbf{j}_{2 p}+\left[\mathbf{A}, \mathbf{j}_{2 p}\right]=0 .
$$

This is also the case in electrodynamics: the minimal coupling is gauge invariant provided the electric charge is conserved. Here, the current has been taken as an external source, whose dynamics is not determined by the action principle. Nevertheless, is the current $\mathbf{j}_{2 p}$ results from particles or fields whose dynamics is governed by an action invariant under the same gauge group $\mathbb{G}$, then its conservation is guaranteed by consistency and one could confirm $D \mathbf{j}_{2 p}=0$ by direct computation, using the explicit form of the current.

An interesting -and possibly the simplest- example of such embedded brane occurs when an identification is made in the spatial slice of $\mathrm{AdS}_{3}$, using a rotational Killing vector that leaves the origin fixed. In that case, a deficit angle is produced and the conical geometry produced around the singularity can be identified with a point particle [37] and the singularity is the worldline of the particle where the curvature behaves like a delta function. The geometry is analogous to that of the BTZ black hole [20,21], but the naked singularity results from a wrong sign in the mass parameter of the solution: $m<0$ [38]. A similar situation arises also when one considers a co-dimension 2 brane in higher dimensions [39]. In all these cases it is confirmed that the coupling between this 0-brane and the (nonabelian) connection is indeed of the form (3.1).

In all these cases, the geometry is only affected in its topological structure, but the local geometry outside the worldline of the source. If the gravity action is invariant under the corresponding $S O(D-1,2)$ gauge group, this symmetry is still respected on any open set outside the worldline. On the worldvolume, on the other hand, the gauge symmetry is reduced to the subgroup of $G$ that commutes with the current.

\section{1 $\theta$-conductors}

The three-dimensional CS action for the $U(1)$ connection (electromagnetic field in $2+1$ dimensions) has a natural interpretation [40]. Consider an electromagnetic field in four-dimensional spacetime, $M$, in which there is a region $\tilde{M}$ occupied by some material characterized by some parameter $\theta$. The action reads

$$
I[A]=\frac{1}{2} \int_{M} F \wedge * F-\frac{\theta}{2} \int_{\tilde{M}} F \wedge F .
$$


The first term is the usual Maxwell action, while the second is $\theta P_{4}$, integrated over the region containing the $\theta$-conductor. This last term can also be written as an integral over the entire spacetime with the help of the characteristic function

$$
\Theta(x)=\left\{\begin{array}{l}
\theta, x \in \tilde{M} \\
0, x \notin \tilde{M}
\end{array} .\right.
$$

Then, the action reads

$$
I[A]=\int_{M}\left(\frac{1}{2} F \wedge * F-\frac{\Theta}{2} F \wedge F\right)
$$

Since $P_{4}$ is a closed form, it does not affect the field equations either inside or outside $M$, and can be locally written as a boundary term,

$$
\begin{aligned}
\int_{M} \frac{\Theta}{2} F \wedge F & =\frac{\theta}{2} \int_{\tilde{M}} F \wedge F \\
& =\frac{\theta}{2} \int_{\tilde{M}} d(A \wedge d A) \\
& =\theta \int_{\partial \tilde{M}} A \wedge d A
\end{aligned}
$$

This can also be written as coupling between the Chern-Simons and a surface current,

$$
\int \frac{\Theta}{2} F \wedge F=\int j \wedge A \wedge d A
$$

Here the surface current is the one-form $j=\theta \delta(\Sigma) d z=d \Theta$, where $z$ is the outward normal coordinate to the surface of $M, \Sigma=\partial M$.

Due to the topological nature of the $\theta$-term, the field equations are the same as those in vacuum. However, this modifies the behavior of the field at the surface $\partial M$. In fact, varying the action (3.6) yields

$$
d * F=j \wedge F
$$

or, in more familiar notation,

$$
\partial_{\mu} F^{\mu \alpha}=\frac{\theta}{2} \delta(\Sigma) \varepsilon^{n \alpha \mu v} F_{\mu v}
$$

where the index $n$ refers to the normal direction to $\Sigma$.

The effect of the $\theta$-term is to introduce an effective current density on the surface of the region $M$. The peculiar feature of this source term is that it is proportional to the components of the electromagnetic field itself, and therefore the electromagnetic superposition principle (linearity of the equations) still holds. Writing (3.8) in coordinates adapted to the surface, one finds ${ }^{3}$

$$
\begin{aligned}
\nabla \cdot \mathbf{E} & =\theta \delta(\Sigma) \mathbf{B} \cdot \mathbf{n} \\
-\partial_{t} \mathbf{E}+\nabla \times \mathbf{B} & =\theta \delta(\Sigma) \mathbf{E} \times \mathbf{n}
\end{aligned}
$$

where $\mathbf{n}$ is the unit normal to $\Sigma$. In the steady state or static case $\left(\partial_{t} \sim 0\right)$, in the vicinity of the surface $\Sigma$ these equations imply that the normal and the tangential components of $\mathbf{E}$ and $\mathbf{B}$,

${ }^{3}$ Here $(\vec{E})_{i}=F^{o i}=-F_{0 i}$ and $(\vec{B}) i=\frac{1}{2} \varepsilon_{i j k} F^{j k}$. 
respectively, are discontinuous,

$$
\begin{aligned}
& {\left[\mathbf{E}_{n}\right]=\theta \mathbf{B}_{n}} \\
& {\left[\mathbf{B}_{\|}\right]=-\theta \mathbf{E}_{\|}}
\end{aligned}
$$

On the other hand, from the Bianchi-Jacobi identity $d F \equiv 0\left(\nabla \cdot \mathbf{B}=0, \partial_{t} \mathbf{B}+\nabla \times \mathbf{E}=0\right)$, it follows that the normal component of $\mathbf{B}$ and the tangential component of $\mathbf{E}$ must be continuous (in the static case at least),

$$
\begin{aligned}
{\left[\mathbf{B}_{n}\right] } & =0 \\
{\left[\mathbf{E}_{\|}\right] } & =0
\end{aligned}
$$

These continuity conditions imply that the right hand sides of (3.11) and (3.12) are well defined and they represent surface charge and current densities, respectively. The phenomenological novelty here is that these sources are given by components of the electromagnetic field itself. The surface charge is proportional to the normal component of the magnetic field, which is similar to the behavior of vortices with magnetic flux as carriers of electric charge in superconductors.

\subsection{Other applications: BCS superconductivity}

Applications of Chern-Simons theories include relevant problems such as the fractional quantum Hall effect and high- $T_{c}$ superconductivity, where nontrivial topology of these effective 2D systems play the key role $[10,11] .^{4}$

The previous example is especially interesting for a general discussion about the phenomenon of superconductivity, even if viewed as a toy model of a superconducting material. Beyond that, it is interesting to mention the connection discovered between a Chern-Simons theory and the BCS theory applied to superconductivity in nanoscopic metallic grains ${ }^{5}$, where a discrete excitation spectrum was shown to exist [43]. It has been shown that this system permits a theoretical treatment based on an exactly solvable reduced BCS model with a fixed number of pairs, and whose solution was discovered long time ago by Richardson [44]. This reduced BCS model is equivalent to a Chern-Simons theory.

The problem can be simplified to the hard-core bosons Hamiltonian

$$
H=\sum_{n, m}^{N}\left[2 \varepsilon_{n} \delta_{n m}-g\right] b_{n}^{+} b_{m},
$$

where the operators $b^{+}(b)$ create (anihilate) a pair, and satisfy commutation relations of the form $\left[b, b^{+}\right]=\delta_{n m}\left(1-2 b_{n} b_{n}^{+}\right)$and $\left(b^{+}\right)^{2}=0(g$ is a model-dependent coupling constant). This apparently simple system is in fact nontrivial because of the above hard-core conditions for the operators; nevertheless, it is exactly solvable.

\footnotetext{
${ }^{4}$ For a review, see [41].

${ }^{5}$ For a review, see [42].
} 
This Hamiltonian is equivalent to a $X Y$ model with long range couplings, which can be expressed in terms of operators satisfying a $S U(2)$ algebra. In this way, it is possible to show the equivalence between this theory with the following CS action

$$
\begin{aligned}
I\left[A ; g_{1}, g_{2}, \ldots, g_{N} ; \xi,\right) & =\frac{k}{4 \pi} \int\left\langle A \wedge d A+\frac{2}{3} A \wedge A \wedge A\right\rangle \\
& +\sum_{n=1}^{N} \int_{-\infty}^{\infty} d t\left\langle\Lambda_{n} g_{n}^{-1}(t)\left(A_{0}+\frac{d}{d t}\right) g_{n}(t)\right\rangle \\
& -\frac{1}{2 \pi i} \int_{-\infty}^{\infty} d t \oint_{C} d z\left\langle\xi A_{0}(z, t)\right\rangle,
\end{aligned}
$$

where $A_{\mu}$ is the $S U(2)$ gauge field, $g_{n}$ are elements of the gauge group field describing the dynamics of a system of interacting coloured particles (temporal Wilson lines insertions) located at points $z_{1}, \ldots, z_{N}$ of a complex 2D plane, and $\Lambda_{n}$ is its highest weight representation. Here $\xi$ is any element of the complexified $S U(2)$ Lie algebra, and $C$ is a non-self-intersecting closed curve in the complex plane which encloses all point-like insertions $z_{n}$ of colored particles.

The resulting Hamiltonian

$$
H=\frac{1}{2} \sum_{n, m}^{N}\left(2 z_{n} \xi_{n} \delta_{n m}-t_{(n)}^{a} t_{(m)}^{a}\right)
$$

where the $t$ 's form a basis for the $S U(2)$ Lie algebra, is shown to be equivalent to the reduced BCS Hamiltonian up to a factor $1 / g$ and an additive constant.

This equivalence deserves a further analysis. However, the connnection is remarkable, providing an interesting geometrical approach to BCS superconductivity. This result establishes an independent discovery that standard superconductivity is related to the Chern-Simons forms, in this case, in terms of an $S U(2)$ theory. The implications, both theoretical and phenomenological, are currently under study [40].

\section{Quantum mechanics}

Assuming the symplectic form $F_{a b}$ to be nondegenerate (invertible), Feynman's quantization of sum over paths can be applied. As already mentioned, the electromagnetic coupling between point charges and the electromagnetic field, as well as the action for any mechanical system with finite number of degrees of freedom are examples of $(0+1)$-dimensional CS systems. The corresponding quantum theories have no mysteries or inconsistencies and the quantum postulates seem to be realized by the demand that the holonomies in phase space take integer values,

$$
\frac{1}{\hbar} e \oint_{\Gamma} A_{\mu} d z^{\mu}=2 n \pi
$$

which is readily recognized as the Bohr-Sommerfeld quantization condition. Other simple examples of quantum behaviour are provided by the quantization of magnetic flux (holonomy around a magnetic center) as in the Aharonov/Bohm effect) and Dirac's quantization of the product of electric and magnetic charges. In all of these cases, the relevant result takes the form (4.1). For details, see [17]. 
The quantum behavior of the $2+1$ CS systems was analyzed by Witten [8], who showed that CS theories are finite (exactly soluble), and provide a framework to understand the Jones polynomial of knot theory in three-dimensional spaces. CS theories are also related to conformal field theories in 1+1 dimensions, furnishing an example of the AdS/CFT correspondence, conjectured a decade later in the context of string theories. Since there is a vast literature over the past twenty years on this subject, there is no point in repeating it here. For an interesting discussion on the relation between Wilson loops in quantum $2+1$ CS theory and knot theory, see [22].

The assertion about the absence of local degrees of freedom applies to the classical solutions, so one could imagine that there might exist fluctuations around locally flat configurations that could possess a quantum spectrum of small oscillations near a stationary point of the action. However, this possibility doesn't exist: small fluctuations around a classical solution should satisfy

$$
\delta F=d(\delta A)=0,
$$

which means that around a classical configuration these must be closed forms, $\delta A=d$ (something). So, all directions in function space around a classical solution are gauge directions, and hence, contribute nothing to the path integral in a perturbative expansion. The conclusion is that the quantum spectrum of $2+1 \mathrm{CS}$ theories -if any- must be nonperturbative. Following the reasoning of [8], it can be seen that the quantum Hilbert space corresponds to nontrivial holonomies (i.e., Wilson loops) of the connection $\mathbf{A}$. What can be expected in higher dimensions?

For the reasons explained above, almost nothing is known about the full quantum behavior of higher-dimensional CS systems. However, all CS systems possess a vacuum configuration, $\mathbf{A}=0$, which is maximally symmetric, absolutely stable, maximally degenerate and isolated from the rest of the configurations. Around this configuration, the theory has no propagating degrees of freedom, and therefore this point defines a topological field theory, like the entire CS theory in three spacetime dimensions. Indeed, in all dimensions, the perturbations around this configuration are pure gauge, $\delta \mathbf{A}=g^{-1}(x) d g(x)$.

Thus, the quantum spectrum of these theories around $\mathbf{A}=0$ is given by all possible locally flat connections defined by the group elements $g: M^{2 n+1} \rightarrow G$. This set is divided into equivalence classes and the quantization problem must be related to the possibility of classifying all inequivalent $g(x)$. For a pure gauge configuration $\mathbf{A}=g^{-1}(x) d g(x)$, the CS action takes the form of a WZ theory without the kinetic term,

$$
I[g]=\text { const } \times \int_{M^{2 n+1}}\left\langle\left(g^{-1} d g\right)^{2 n+1}\right\rangle,
$$

which defines a field theory for $g$ on the boundary of $M^{2 n+1}$. This boundary theory inherits all the symmetries of the bulk action, has propagating degrees of freedom, and must also contain nonperturbative and nonlocal states, analogous to Wilson loops, but which in this case would be higher dimensional surfaces.

\section{Summary}

CS systems are quite ubiquitous in physics. As we have seen, any mechanical system with a finite number of degrees of freedom and the interaction term between a point charge and an electromagnetic field are $(0+1)$-CS systems. The Bohr-Sommerfeld quantization rules correspond 
to quantization of holonomies of loops in phase space, or of the symplectic flux enclosed by those loops. Dirac's quantization of the product of magnetic and electric charge is a particular case of the same quantization rule, and that same condition arises in the quantization of the only free parameter of Chern-Simons theories in $2 n+1$ dimensions, and in particular, in the quantization of the analogue of Newton's constant in CS gravities [46].

Higher dimensional CS systems define dynamical theories for a connection field. These higher-dimensional actions can also be interpreted as consistent couplings between $2 p$-branes and (non)abelian connections. In both cases there is no need for the background spacetime to be endowed with a metric structure. Thus, CS systems seem better suited to define a more basic underlying framework for the fundamental description of fields and sources.

In closing, let us summarize the features of the functional (2.3) that are common to all CS actions,

- Topological origin. The CS form is related to a topological density in $2 n$ dimensions known as a characteristic class $C_{2 n}$, through

$$
d \mathscr{C}_{2 k-1}(\mathbf{A})=P_{2 k}(\mathbf{A}) .
$$

- No metric required. Since the CS forms are local $(2 k+1)$-forms, they are ready to integrate over $\Gamma^{2 k+1}$, without any additional structure. In particular, the metric of the embedding space, $M^{2 s+1}$, is irrelevant. This is a consequence of the topological origin of the CS forms.

- Gauge quasi-invariance. The functional (2.3) involves explicitly the connection $A$ and cannot be expressed as an integral of a gauge invariant local function. However, under a gauge transformation $A \rightarrow A+d \Omega, I$ changes by the surface term

$$
\delta I=e \int_{\Gamma} d \Omega=e\left[\Omega\left(z_{+}\right)-\Omega\left(z_{-}\right)\right],
$$

where $z_{+}$and $z_{-}$are the end points of the worldline $\Gamma$. Thus, the action is not strictly gauge invariant, but quasi invariant. However, $I$ is a genuine gauge invariant object for the class of gauge transformations that have periodic boundary conditions (no boundary), $\Omega\left(z_{+}\right)=$ $\Omega\left(z_{-}\right)$. Higher-dimensional CS forms are also quasi invariant, and this is sufficient to define a consistent coupling to conserved sources of the form (3.1).

- Nontrivial dynamics. Even though there is no "kinetic term" $\left(\dot{z}^{2} / 2\right)$ in $I$, the lagrangian defined by (2.3) gives a meaningful action principle. Indeed, varying with respect to $z$ one obtains the first order equations

$$
F_{a b} \dot{z}^{b}=0,
$$

which describe the motion of a charge under the influence of an external electromagnetic field such that the electric and magnetic forces exactly cancel each other. Varying with respect to $A$ informs us that this field can only vary as a connection, but is otherwise arbitrary. In higher dimensions, the field $\mathbf{A}$ is not an arbitrary connection without dynamics. The variation of the action with respect to it yields a set of field equations for $\mathbf{A}$ on the $(2 n+1)$-dimensional worldvolume of the brane to which it couples. 


\section{Acknowledgments}

This work was supported by Fondecyt grants \# 1100755, 1100328, and by Conicyt grant Southern Theoretical Physics Laboratory, ACT-91. L. Huerta is partially supported by the P4-Center for Research and Applications in Plasma Physics and Pulsed Power Technology, under grant PBCTChile, ACT-26. The Centro de Estudios Científicos (CECS) is funded by the Chilean Government through the Centers of Excellence Base Financing Program of Conicyt.

\section{References}

[1] M. Nakahara, Geometry, Topology and Physics, (Graduate Student Series in Physics), Taylor \& Francis; 2 edition (2003).

[2] J. Schonfeld, A mass term for three dimensional gauge fields, Nucl. Phys. B185, 157 (1981).

[3] S. Deser, R. Jackiw and S. Templeton, Three dimensional massive gauge theories, Phys. Rev.Lett. 48, 975 (1983); Topologically massive gauge theory. Ann. Phys. NY 140, 372 (1984).

[4] S. S. Chern and J. Simons, Characteristic forms and geometric invariants, Annals Math. 99, 48-69 (1974).

[5] S. Deser, Chern-Simons terms as an example of the relations between mathematics and physics, [arxiv:math-ph/9805020]; and Physico - mathematical interactions: The Chern-Simons story, [arxiv:math-ph/9812005]

[6] E. Cremmer, B. Julia and J. Scherk, Supergravity theory in 11 dimensions, Phys. Lett. B76 (1978) 409.

[7] A. P. Balachandran, L. Chandar and B. Sathiapalan, Chern-Simons duality and the quantum Hall effect, Int. J. Mod. Phys. A 11 (1996) 3587 [arXiv:hep-th/9509019].

[8] E. Witten, Quantum field theory and the Jones polynomial, Commun. Math. Phys. 121, 351 (1989).

[9] M. Bos and V. P. Nair, U(1) Chern-Simons Theory and $c=1$ Conformal Blocks, Phys.Lett. B223 (1989), 61; Coherent State Quantization of Chern-Simons Theory, Int. J. Mod. Phys. A 5 (1990) 959.

S. Elitzur, G. W. Moore, A. Schwimmer and N. Seiberg, Remarks On The Canonical Quantization Of The Chern-Simons-Witten Theory, Nucl. Phys. B 326 (1989) 108. J. M. F. Labastida and A. V. Ramallo, Operator formalism for Chern-Simons theories, Phys. Lett. B 227 (1989) 92.

H. Murayama, Explicit quantization of the Chern-Simons action, Z. Phys. C 48 (1990) 79.

A. P. Polychronakos, Abelian Chern-Simons Theories in (2+1)-dimensions, Annals Phys. 203 (1990) 231. T. R. Ramadas, I. M. Singer and J. Weitsman, Some comments on Chern-Simons gauge theory, Commun. Math. Phys. 126 (1989) 409. G. V. Dunne, R. Jackiw, S. Y. Pi and C. A. Trugenberger, Selfdual Chern-Simons solitons and two-dimensional nonlinear equations, Phys. Rev. D 43 (1991) 1332; [Erratum-ibid. D 45 (1992) 3012].

[10] X. G. Wen, F. Wilczek and A. Zee, Phys. Rev. B39 (1989) 11413.

[11] See A. P. Balachandran, E. Ercolessi, G. Morandi, A.M. Srivastava, Hubbard Model and Anyon Superconductivity, World Scientific Publishing Co. Inc. (1990) 100-108

[12] A. Achúcarro and P. K. Townsend, A Chern-Simons action for three-dimensional anti-De Sitter supergravity Theories, Phys. Lett. B180 (1986) 89.

[13] A. Achúcarro and P. K. Townsend, Extended supergravities in $d=(2+1)$ as Chern-Simons theories, Phys. Lett. B229 (1989) 383.

[14] E. Witten, (2+1)-Dimensional gravity as an exactly soluble system, Nucl. Phys. B311 (1988) 46. 
[15] A. H. Chamseddine, Topological gravity and supergravity in various dimensions, Nucl. Phys. B346 (1990) 213.

[16] J. Zanelli, Lecture notes on Chern-Simons (super-)gravities. [arXiv:hep-th/0502193].

[17] J. Zanelli, Uses of Chern-Simons actions, Talk given at Ten Years of AdS/CFT: A Workshop Celebrating the Tenth Anniversary of the Maldacena Conjecture, Buenos Aires, Argentina, Dec. 2007, AIP Conf. Proc. 1031, 115 (2008)[arXiv:0805.1778 [hep-th]].

[18] J. Saavedra, R. Troncoso and J. Zanelli, Chern-Simons in $0+1 D$ and its dynamic singular properties, Rev. Mex. Fis. 48 (2002) SUPPL387; Degenerate dynamical systems, J. Math. Phys. 42 (2001) 4383, [arXiv:hep-th/0011231].

[19] F. Dyson, Feynman's proof of the Maxwell equations, Am.J.Phys. 58 (1990) 209-211.

[20] M. Bañados, C. Teitelboim and J. Zanelli, The Black hole in three-dimensional space-time, Phys. Rev. Lett. 69 (1992) 1849. [arXiv:hep-th/9204099].

[21] M. Bañados, M. Henneaux, C. Teitelboim and J. Zanelli, Geometry of the (2+1) black hole, Phys. Rev. D48 (1993) 1506. [arXiv:gr-qc/9302012].

[22] J. M. F. Labastida and M. Marino, Topological quantum field theory and four manifolds, Dordrecht, Netherlands: Springer (2005); J. M. F. Labastida, Chern-Simons Gauge Theory: Ten years After, AIP Conference Proceedings, vol. 484, 1999. [arXiv:hep-th/9905057]

[23] A. Zee, Quantum Field Theory in a Nutshell: Second Edition, Princeton University Press; 2nd edition (2010).

[24] V. Nair, Quantum Field Theory: A Modern Perspective, Graduate Texts in Contemporary Physics, Springer (2010)

[25] A. Mardones and J. Zanelli, Lovelock-Cartan theory of gravity, Class. Quant. Grav. 8, 1545 (1991).

[26] R. Floreanini and R. Percacci, Higher Dimensional Abelian Chern-Simons Theory, Phys. Lett. B 224 (1989) 291; Four-dimensional current algebra from Chern-Simons Theory, Phys. Lett. 231 (1989) 119.

[27] V. P. Nair and J. Schiff, A Kahler-Chern-Simons theory and quantization of instanton moduli spaces, Phys. Lett. 246 (1990) 423; Kahler Chern-Simons theory and symmetries of antiselfdual gauge fields, Nucl. Phys. 371 (1992) 329.

[28] M. Bañados, L. J. Garay and M. Henneaux, The local degrees of freedom of higher dimensional pure Chern-Simons theories, Phys. Rev. D 53 (1996) 593, [arXiv:hep-th/9506187]; The dynamical structure of higher dimensional Chern-Simons theory, Nucl. Phys. B 476 (1996) 611, [arXiv:hep-th/9605159].

[29] P.A.M.Dirac, Lectures on Quantum Mechanics, Dover Publications, N.Y., 2001; Proc. Roy. Soc. A133 (1931) 60

[30] O. Mišković and J. Zanelli, Irregular hamiltonian systems, Proceedings of "XIII Chilean Symposium of Physics", (Concepción, Chile, 2002). [arXiv:hep-th/0301256].

[31] O. Mišković and J. Zanelli, Dynamical structure of irregular constrained systems, J. Math. Phys. 44 (2003) 3876. [arXiv:hep-th/0302033].

[32] M. Bañados, R. Troncoso and J. Zanelli, Higher dimensional Chern-Simons supergravity, Phys. Rev. D 54, 2605 (1996). [arXiv:gr-qc/9601003]. 
[33] R. Troncoso and J. Zanelli, New gauge supergravity in seven and eleven dimensions, Phys. Rev. D 58, 101703 (1998). [arXiv:hep-th/9710180]; Gauge supergravities for all odd dimensions, Int. J. Theor. Phys. 38, 1181 (1999). [arXiv:hep-th/9807029].

[34] C. Teitelboim, Gauge invariance for extended objects, Phys. Lett. B 167, 63 (1986). M. Henneaux and C. Teitelboim, P-Form Electrodynamics, Found. Phys. 16, 593 (1986).

[35] J. D. Edelstein and J. Zanelli, Sources for Chern-Simons theories, in "Quantum Mechanics of Fundamental Systems: The Quest for Beauty and Simplicity", M. Henneaux and J. Zanelli (eds.) (Springer-Verlag, New York), pp. 107-124 (2009).arXiv:0807.4217 [hep-th].

[36] J. D. Edelstein, A. Garbarz, O. Mišković and J. Zanelli, Naked singularities, topological defects and brane couplings. To appear in "Quantum Gravity and the Foundations of Physics" Conference in honor of Prof. Mario Castagnino's 75th birthday, Rosario, Argentina, Mar 2010. arXiv:1009.4418 [hep-th].

[37] S. Deser, R. Jackiw and G. 't Hooft, Three-Dimensional Einstein Gravity: Dynamics Of Flat Space, Annals Phys. 152, 220 (1984). S. Deser and R. Jackiw, Three-Dimensional Cosmological Gravity: Dynamics Of Constant Curvature, Annals Phys. 153, 405 (1984); String sources in $(2+1)$-dimensional gravity, Annals Phys. 192, 352 (1989)

[38] O. Mišković and J. Zanelli, On the negative spectrum of the 2+1 black hole, Phys. Rev. D 79, 105011 (2009). [arXiv:0904.0475 [hep-th]].

[39] O. Mišković and J. Zanelli, Couplings between Chern-Simons gravities and 2p-branes, Phys. Rev. D 80, 044003 (2009). [arXiv:0901.0737 [hep-th]].

[40] F. Canfora, L. Huerta, L. La Rosa and J. Zanelli, work in progress.

[41] E. Fradkin, Field Theories of Condensed Matter Systems, Addisson Wewsley Co. (1991) 241-249.

[42] J. von Delft, and D.C. Ralph, Spectroscopy of discrete energy levels in ultrasmall metallic grains, Phys. Rep. 345 (2001) 61-173.

[43] M. Asorey, F. Falceto and G. Sierra, Chern-Simons theory and BCS superconductivity, Nucl. Phys. B 622, 593 (2002) [arXiv:hep-th/0110266].

[44] R.W. Richardson, Phys. Lett. 3, 277 (1963). R.W. Richardson, Phys. Lett. 5, 82 (1963). R.W. Richardson and N. Sherman, Nucl. Phys. B 52, (1964) 221. R.W. Richardson, J. Math. Phys. 6, 1034 (1965)

[45] P. A. M. Dirac, Quantised singularities in the electromagnetic field, Proc. Roy. Soc. Lond. A 133, 60 (1931).

[46] J. Zanelli, Quantization of the gravitational constant In odd-dimensional gravity, Phys. Rev. D 51, 490 (1995). [arXiv:hep-th/9406202].

[47] J. D. Edelstein, A. Garbarz, O. Mišković and J. Zanelli, Stable p-branes in Chern-Simons AdS supergravities, Phys. Rev. D 82, 044053 (2010). [arXiv:1006.3753 [hep-th]]. 